\title{
OPTIMIZACIÓN DE LA DESHIDRATACIÓN Y EXTRACCIÓN DE COMPUESTOS FENÓLICOS DE PIEL DE MANGO
}

\author{
Nicole Febres Burneo ${ }^{a}$, María Mora-Medina ${ }^{a}$ y Jorge G. Figueroa ${ }^{\mathrm{a}, *,(1)}$ \\ a'Departamento de Química, Universidad Técnica Particular de Loja, 11-01-608 Loja - Loja, Ecuador
}

Recebido em 10/08/2021; aceito em 20/10/2021; publicado na web em 18/11/2021

\begin{abstract}
OPTIMIZATION OF DEHYDRATION AND EXTRACTION OF PHENOLIC COMPOUNDS FROM MANGO PEEL. Mango peel, a by-product obtained from the industrialization of this fruit, is a promising and inexpensive source of phenolic compounds. In the present study, both the drying of the mango peel and the extraction of phenolic compounds were optimized using the response surface methodology. In this sense, temperature and charge/area ratio were selected as depend variables to drying process. Instead, for the ultrasonic assisted extraction (UAE) the temperature, ethanol-water concentration and time were optimized. For both processes, the total phenolic content (TPC) and mangiferin concentrations were the response variables. The TPC and the xanthone concentration were determined by the Folin-Ciocalteu method and HPLC-ESI-IT-MS ${ }^{\mathrm{n}}$, respectively. The optimal conditions for the dehydration process were $54{ }^{\circ} \mathrm{C}$ and $2.3 \mathrm{~kg} \mathrm{~m}^{-2}$, reaching a TPC of $21.7 \pm 1.6 \mathrm{mg} \mathrm{GAE} \mathrm{g}^{-1} \mathrm{dw}$ and a concentration of mangiferin of $1.94 \pm 0.22 \mathrm{mg} \mathrm{g}^{-1} \mathrm{dw}$. Regarding to UAE, the optimum conditions were $51{ }^{\circ} \mathrm{C}, 50 \%$ ethanol and $71 \mathrm{~min}$. Under these conditions, the TPC was $19.5 \pm 0.8 \mathrm{mg} \mathrm{GAE} \mathrm{g}^{-1} \mathrm{dw}$ and the mangiferin concentration corresponded to $1.50 \pm 0.13 \mathrm{mg} \mathrm{g}^{-1} \mathrm{dw}$. Additionally, 38 compounds were identified in the extract obtained under optimal conditions.
\end{abstract}

Keywords: by-product; Mangifera indica L.; mangiferin; polyphenols; response surface methodology.

\section{INTRODUCCIÓN}

El Mango es la fruta de mayor importancia económica en la familia Anacardiaceae, ${ }^{1}$ y debido a sus inigualables cualidades organolépticas y su elevado valor nutricional se lo ha denominado "El Rey de las frutas". ${ }^{2}$ Por muchos años, la atención de las industrias se ha centrado en la pulpa, convirtiéndose en la base de un sinnúmero de productos tales como: jugo, néctar, rebanadas de mango en almíbar, formulaciones para comida de bebe, saborizante de productos lácteos, etc. ${ }^{3}$ Sin embargo, la producción de estos alimentos genera grandes toneladas de subproductos, principalmente piel y semilla, que, al ser desechados como residuos, representan una fuente de contaminación ambiental. ${ }^{4}$ Actualmente, existen evidencias que demuestran que estos subproductos al ser ricos en compuestos fenólicos, pueden ser reutilizados como ingredientes en la formulación de alimentos funcionales y nutracéuticos, gracias a los múltiples beneficios que estos aportan a la salud humana. ${ }^{5}$ Ahora bien, el alto contenido de agua presente en la piel de mango, hace que este subproducto sea susceptible a descomposición durante el almacenamiento, ${ }^{6}$ siendo la deshidratación, una técnica clave para disminuir la actividad de agua a un nivel que disminuya las reacciones de deterioro. ${ }^{7}$

Por otro lado, las altas temperaturas y los tiempos prolongados asociados a los métodos convencionales de extracción como la maceración sólido-líquido y Soxhlet, han estimulado la búsqueda de nuevas técnicas que sean compatibles con los requerimientos de la industria alimentaria y farmacéutica, es decir, métodos sostenibles, menos tóxicos y que generan un impacto ambiental positivo. ${ }^{8} \mathrm{La}$ extracción asistida por ultrasonido (UAE) emerge como una excelente alternativa a los métodos tradicionales gracias a su simplicidad y eficiencia. ${ }^{9}$ Además, permite obtener una alta reproducibilidad en menor tiempo, reduce significativamente en el consumo de solvente, y tiene un menor aporte energético. ${ }^{10}$

Sin embargo, es necesario optimizar los parámetros para realizar una UAE exitosa. En este sentido, la metodología de superficie de

\footnotetext{
*e-mail: jgfigueroa@utpl.edu.ec
}

respuesta es considerada como una herramienta estadística exitosa cuando se desea optimizar un proceso. ${ }^{11}$ La principal ventaja de este método es que, al reducir los ensayos experimentales, es menos laboriosa y, por lo tanto, requiere menos tiempo que otros enfoques utilizados para optimizar procesos. ${ }^{12}$

Teniendo en cuenta las razones antes mencionadas, el presente estudio buscó optimizar los procesos de deshidratación y extracción de compuestos fenólicos de piel de mango por la técnica UAE, que permitan obtener un extracto rico en antioxidantes.

\section{PARTE EXPERIMENTAL}

\section{Reactivos}

Para la extracción de compuestos fenólicos se utilizó etanol, acetona y ácido clorhídrico suministrados por Merck KGaA (Darmstadt, Alemania) y metanol proveniente de Pareack Applichem (Barcelona, España). Los reactivos utilizados en la cuantificación de fenoles totales: reactivo Folin Ciocalteu, ácido gálico y carbonato de sodio fueron obtenidos de Merck KGaA (Darmstadt, Alemania). Por otra parte, para la identificación del perfil fenólico y optimización de mangiferina por HPLC se utilizó acetonitrilo (ACN) grado HPLC procedente de Merck KGaA (Darmstadt, Alemania) y ácido acético obtenido de J.T. Baker Analyzed. Se utilizó el estándar de mangiferina suministrado por Sigma-Aldrich (Misuri, USA).

\section{Muestras}

En la presente investigación se trabajó con mango (Mangifera indica L. cv. "Tommy Atkins"), adquirido en un local comercial del catón Catamayo, provincia de Loja, Ecuador. La muestra se lavó y luego se sometió a un proceso de escaldado a $75{ }^{\circ} \mathrm{C}$ por 8 min con el fin de reducir la actividad enzimática. ${ }^{13}$ Posteriormente, se separó mecánicamente la piel del resto de la fruta e inmediatamente fueron deshidratadas. El estado de madurez de la fruta fue evaluado mediante el análisis de sólidos solubles y acidez titulable según los métodos 
AOAC 932.12 y AOAC 942.15, respectivamente. El contenido de humedad se determinó siguiendo el protocolo descrito en el método AOAC 934.06. ${ }^{14}$

\section{Optimización del proceso de deshidratación}

Se optimizó el proceso de deshidratación utilizando metodología de superficie de respuesta (MSR). Para ello, se empleó un diseño central compuesto (DCC), debido a que permite comprender las interacciones entre los parámetros que se van a optimizar. ${ }^{15}$ Las variables seleccionadas fueron: temperatura $\left(40-70{ }^{\circ} \mathrm{C}\right)$ y relación carga/área (1,80-3 $\left.\mathrm{kg} \mathrm{m}^{-2}\right)$, mientras que el contenido de fenoles totales y mangiferina fueron las variables de respuesta seleccionadas. Los diferentes experimentos se llevaron a cabo de forma aleatoria, para minimizar los efectos de la variabilidad en las respuestas observadas.

La deshidratación de la piel de mango se realizó hasta obtener un contenido de humedad menor al $10 \%$ y una actividad de agua entre 0.3 y 0.4. Esta operación se llevó a cabo en un deshidratador tipo bandejas con circulación de aire caliente (DY-110H, Lassele, Korea). Las condiciones ensayadas se detallan en la Tabla 1. Además, como control del proceso, se deshidrató la piel de mango en un liofilizador (7754047, Labconco, USA). Posteriormente, las muestras deshidratadas se trituraron en un molino ultracentrífugo hasta un tamaño de partícula $<500 \mu \mathrm{m}$ (ZM 200, RTSCH, Alemania), dimensión que facilita la extracción de los compuestos fenólicos. ${ }^{16}$

Tabla 1. Diseño Central Compuesto para la optimización del proceso de deshidratación

\begin{tabular}{lcccc}
\hline Experimento & $\begin{array}{c}\text { Temperatura } \\
\left({ }^{\circ} \mathrm{C}\right)\end{array}$ & $\begin{array}{c}\text { Carga/Área } \\
\left(\mathrm{kg} \mathrm{m}^{-2}\right)\end{array}$ & $\begin{array}{c}\text { TPC } \\
\left(\mathrm{mg} \mathrm{GAE} \mathrm{g}^{-1} \mathrm{bs}\right)\end{array}$ & $\begin{array}{c}\text { Mangiferina } \\
\left(\mathrm{mg} \mathrm{g}^{-1} \mathrm{bs}\right)\end{array}$ \\
\hline 1 & 55 & 3.0 & 19.4 & 0.94 \\
2 & 40 & 2.4 & 20.6 & 1.39 \\
3 & 66 & 2.8 & 19.8 & 1.31 \\
4 & 55 & 2.4 & 22.4 & 1.95 \\
5 & 44 & 2.0 & 20.8 & 1.54 \\
6 & 55 & 2.4 & 21.9 & 1.81 \\
7 & 44 & 2.8 & 20.6 & 1.33 \\
8 & 55 & 2.4 & 22.5 & 1.84 \\
9 & 55 & 2.4 & 21.6 & 1.80 \\
10 & 55 & 2.4 & 22.2 & 1.80 \\
11 & 66 & 2.0 & 21.4 & 1.38 \\
12 & 55 & 1.8 & 20.7 & 1.12 \\
13 & 70 & 2.4 & 21.6 & 1.31 \\
\hline \multicolumn{5}{c}{ Condiciones Óptimas } \\
\hline Modelo & 54 & 2.3 & 22.1 & 1.84 \\
Experimental & & \multicolumn{5}{c}{$1.94 \pm 0.22$} \\
\hline
\end{tabular}

La extracción de compuestos fenólicos se realizó de acuerdo al protocolo descrito por Pérez-Jiménez y Saura-Calixto ${ }^{17}$ Brevemente, se pesó $1 \mathrm{~g}$ de muestra en un tubo de centrífuga y se adicionó $20 \mathrm{~mL}$ de una disolución metanol-agua (50:50 v/v, pH 2 acidificados con $\mathrm{HCl}$ ). Se extrajo durante 1 hora a $200 \mathrm{rpm}$ empleando un agitador horizontal (3018, GFL) y luego se realizó la separación de fases utilizando la centrifuga (Sorvall ST8, ThermoFisher Scientific, USA) a $8700 \mathrm{rpm}$ por 30 min. Posteriormente, se recuperó el sobrenadante y, al residuo se le agregó $20 \mathrm{~mL}$ de acetona: agua (70:30 v/v), al cual nuevamente se lo agitó y se centrifugó hasta obtener un nuevo sobrenadante. Finalmente, los sobrenadantes se combinaron y almacenaron a $-20{ }^{\circ} \mathrm{C}$ hasta su posterior análisis.

\section{Optimización del proceso de extracción}

Una vez optimizado la operación de deshidratación, se optimizó el proceso de extracción de compuestos fenólicos de piel de mango. Como técnica de extracción se seleccionó la extracción asistida por ultrasonidos. Las variables independientes estudiadas fueron, temperatura de extracción $\left(25-60{ }^{\circ} \mathrm{C}\right)$, relación etanol-agua $(0-100 \%)$ y el tiempo de extracción (5-120 min). Las variables dependientes seleccionadas fueron el contenido de fenoles totales y mangiferina. Se utilizó el diseño Box-Behnken (DBB), metodología que permite una optimización rápida, económica y eficiente cuando se desea estudiar los efectos de tres o más variables. ${ }^{18}$

Las extracciones se llevaron a cabo en un baño de ultrasonidos (FS30D, Fisher Scientific, USA) con una frecuencia de $42 \mathrm{kHz}$ y a $100 \mathrm{~W}$ de potencia. En un tubo de centrífuga se pesó $1 \mathrm{~g}$ de muestra y se agregó $20 \mathrm{~mL}$ del solvente de acuerdo con las concentraciones indicadas en la Tabla 2. Posteriormente, las muestras se extrajeron con las diferentes temperaturas y tiempos indicados por el DBB e inmediatamente después se centrifugaron a $8700 \mathrm{rpm}$ durante 30 min. Tras la centrifugación, los sobrenadantes se recogieron para la determinación de fenoles totales y mangiferina.

\section{Determinación del contenido de fenoles totales}

Para determinar el contenido de fenoles totales se utilizó el método colorimétrico de Folin-Cioacalteu descrito por Figueroa et al. ${ }^{7}$ Se utilizó ácido gálico para construir la curva de calibración. Los resultados obtenidos se expresaron como miligramos equivalentes de ácido gálico por gramo de muestra en base seca (mg EAG g ${ }^{-1}$ base seca).

\section{Cuantificación de mangiferina por HPLC-ESI-IT-MS ${ }^{\text {n }}$}

Para el análisis de mangiferina se utilizó un HPLC (Dionex Ultimate 3000, ThermoScientific, USA) acoplado a un espectrómetro de masas de trampa de iones equipado con una unidad de ionización por electrospray (ESI) (Amazon Speed, Bruker Daltonics, Bremen, Alemania). La separación se realizó utilizando una columna C18 de dimensiones $150 \mathrm{~mm}$ x 3,0 mm x $3 \mu \mathrm{m}$ (Sunfire, Waters, USA). Las condiciones se fijaron de acuerdo a los descrito por Gómez-Caravaca et al. ${ }^{19}$ Brevemente, la fase móvil A consistió en agua con $0,1 \%$ de ácido acético v/v, y la fase móvil B fue acetonitrilo. Las condiciones de elución fueron: $0 \mathrm{~min}, 0 \% \mathrm{~B} ; 5.5 \mathrm{~min}, 6.8 \% \mathrm{~B}$; $16 \mathrm{~min}, 20 \% \mathrm{~B} ; 20 \mathrm{~min}, 25 \% \mathrm{~B} ; 25 \mathrm{~min}, 35 \% \mathrm{~B} ; 29 \min , 100 \% \mathrm{~B}$; $36 \mathrm{~min}, 100 \% \mathrm{~B} ; 38 \mathrm{~min}, 0 \% \mathrm{~B} ; 40 \mathrm{~min}, 0 \% \mathrm{~B}$. El volumen de inyección fue de $10 \mu \mathrm{L}$ y el caudal se ajustó a $0,4 \mathrm{~mL} \min ^{-1}$. La columna fue mantenida a $30^{\circ} \mathrm{C}$.

$\mathrm{El}$ análisis espectrométrico de masas se realizó en modo ultrascan. Los parámetros de adquisición fueron los siguientes: polaridad iónica: negativa; presión del nebulizador: 6 psi; flujo de secado del gas $\left(\mathrm{N}_{2}\right)$ : $6 \mathrm{~L} \mathrm{~min}{ }^{-1}$; temperatura de secado: $200{ }^{\circ} \mathrm{C}$; voltaje capilar: $3500 \mathrm{~V}$; desplazamiento de la placa final: $500 \mathrm{~V}$ y rango de escaneado: 100$1000 \mathrm{~m} / \mathrm{z}$. Los datos se recopilaron y procesaron utilizando el software Compass DataAnalysis.

\section{RESULTADOS Y DISCUSIÓN}

El estado de madurez de las frutas tiene efecto sobre el contenido de compuestos fenólicos. ${ }^{20}$ En este sentido, previo a la optimización de los procesos de deshidratación y extracción, se evaluó el grado de madurez de la fruta utilizada. Indudablemente, la relación ${ }^{\circ}$ Brix/acidez es un indicador confiable para estudiar este parámetro en 
Tabla 2. Diseño Box-Behnken para la optimización de la extracción de compuestos fenólicos

\begin{tabular}{|c|c|c|c|c|c|}
\hline Experimento & $\begin{array}{c}\mathrm{EtOH} \\
(\%)\end{array}$ & $\begin{array}{l}\text { Time } \\
(\mathrm{min})\end{array}$ & $\begin{array}{c}\text { Temperatura } \\
\left({ }^{\circ} \mathrm{C}\right)\end{array}$ & 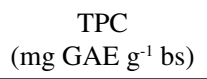 & $\begin{array}{c}\text { Mangiferina } \\
\left(\mathrm{mg} \mathrm{g}^{-1} \mathrm{bs}\right)\end{array}$ \\
\hline 1 & 50 & 120.0 & 25.0 & 8.9 & 0.90 \\
\hline 2 & 50 & 62.5 & 42.5 & 20.0 & 1.20 \\
\hline 3 & 100 & 120.0 & 42.5 & 6.5 & 0.60 \\
\hline 4 & 50 & 5.0 & 60.0 & 11.2 & 1.00 \\
\hline 5 & 0 & 62.5 & 25.0 & 7.6 & 0.60 \\
\hline 6 & 0 & 120.0 & 42.5 & 5.9 & 0.60 \\
\hline 7 & 50 & 120.0 & 60.0 & 16.0 & 1.10 \\
\hline 8 & 100 & 5.0 & 42.5 & 2.3 & 0.40 \\
\hline 9 & 100 & 62.5 & 60.0 & 9.8 & 0.70 \\
\hline 10 & 50 & 62.5 & 42.5 & 19.8 & 1.20 \\
\hline 11 & 50 & 5.0 & 25.0 & 14.3 & 0.90 \\
\hline 12 & 0 & 62.5 & 60.0 & 5.6 & 0.70 \\
\hline 13 & 100 & 62.5 & 25.0 & 4.8 & 0.50 \\
\hline 14 & 0 & 5.0 & 42.5 & 5.7 & 0.60 \\
\hline 15 & 50 & 62.5 & 42.5 & 18.3 & 1.20 \\
\hline \multicolumn{6}{|c|}{ Condiciones óptimas } \\
\hline Modelo & \multirow{2}{*}{50} & \multirow{2}{*}{71.0} & \multirow{2}{*}{51.0} & 19.37 & 1.22 \\
\hline Experimental & & & & $19.5 \pm 0.8$ & $1.50 \pm 0.13$ \\
\hline
\end{tabular}

matrices vegetales. De los análisis realizados, en el presente estudio se pudo determinar una relación ${ }^{\circ}$ Brix/acidez de $22.1 \pm 0.4$, lo que confirma que la materia prima presentó un estado de madurez óptimo para su consumo. ${ }^{21}$

\section{Optimización del proceso de deshidratación}

El contenido de humedad inicial de la piel de mango fue $80,73 \pm 1,30 \%$, valor cercano al reportado por Sogi et al.,$^{5} \mathrm{de}$ $83,37 \%$. Debido a este elevado contenido, fue imprescindible realizar un proceso de deshidratación para reducir este contenido a valores inferiores a $10 \%$, con el propósito de evitar el deterioro por microorganismos y retardar procesos enzimáticos propios del subproducto. ${ }^{13}$ Además, se conoce que la piel de mango es rica en compuestos fenólicos. Por lo tanto, con la finalidad de reducir el deterioro de este tipo de compuestos durante la deshidratación, es necesario optimizar el proceso de deshidratación. En esta investigación, se seleccionó la temperatura de deshidratación y

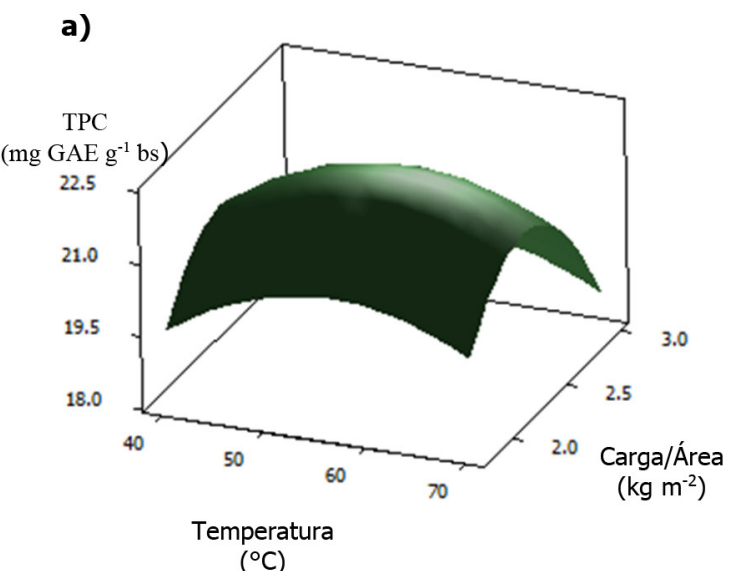

la relación carga/área como variables independientes. Los rangos evaluados se fijaron en base a estudios previos. Respecto a la temperatura, el valor superior $\left(70^{\circ} \mathrm{C}\right)$ estuvo limitado por la capacidad del deshidratador, mientras que, para la selección del límite inferior $\left(40{ }^{\circ} \mathrm{C}\right)$, se consideró que, a temperaturas bajas, se necesitan tiempos más prolongados de deshidratación, lo que conlleva a una degradación de los compuestos fenólicos. ${ }^{7}$ Con respecto a la relación carga/área, el rango seleccionado estuvo en función al utilizado en el estudio realizado por Saavedra et al., ${ }^{22}$ quienes optimizaron el proceso de deshidratación de los subproductos de aguacate (piel y semilla).

Luego del proceso de deshidratación, el TPC y la concentración de mangiferina variaron desde 19.4 hasta $22.5 \mathrm{mg} \mathrm{EAG} \mathrm{g}^{-1} \mathrm{de}$ piel de mango y desde 0.94 hasta $1.95 \mathrm{mg} \mathrm{g}^{-1}$ de piel de mango, respectivamente. En las Figuras $1 \mathrm{a}$ y b, se ilustra el efecto de la temperatura y la relación carga/área sobre el TPC y la concentración de mangiferina, respectivamente. Como se puede apreciar, existe un aumento en las variables respuesta analizadas cuando las temperaturas de deshidratación son cercanas a $55^{\circ} \mathrm{C}$ y la relación

b)

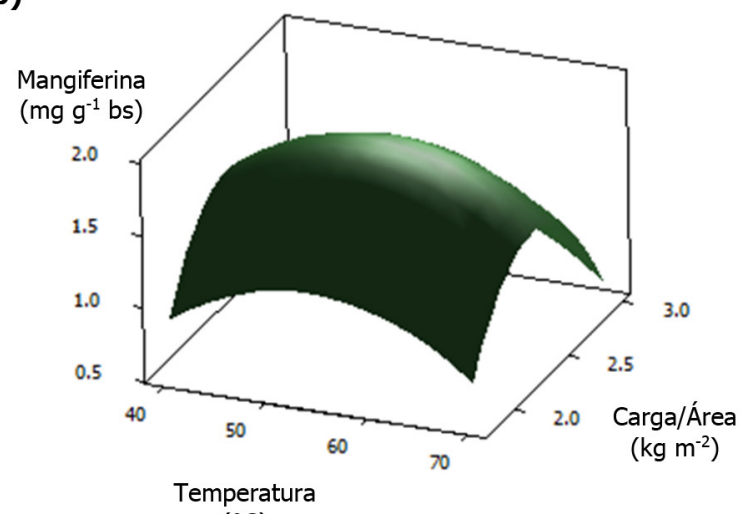

$\left({ }^{\circ} \mathrm{C}\right)$

Figura 1. Gráficos de superficie de respuesta que muestran el efecto de la temperatura de deshidratación y relación carga área sobre: a) TPC y b) concentración de mangiferina 
carga/área es aproximadamente $2.5 \mathrm{~kg} \mathrm{~m}^{-2}$ (Figura 1). Sin embargo, superado estos límites se produce un descenso notorio en las variables respuesta. Esto se debe principalmente, a la degradación térmica de los compuestos fenólicos presentes en la muestra, ${ }^{23}$ y a la unión de estos compuestos a estructuras proteicas. ${ }^{24}$ Miranda et al. ${ }^{25}$ deshidrataron semillas de quinua utilizando diferentes condiciones de temperatura $\left(40-80{ }^{\circ} \mathrm{C}\right)$, y encontraron una reducción en el contenido fenólico cuando a valores superiores a $60{ }^{\circ} \mathrm{C}$, resultado similar al que se obtuvo en el presente trabajo. Además, Saavedra et al., ${ }^{22}$ evaluaron el efecto de la temperatura, carga/área y velocidad de aire sobre el contenido fenólico de la piel y semilla de aguacate, y observaron un contenido máximo de fenoles totales cuando la relación carga/área es $2 \mathrm{~kg} \mathrm{~m}^{-2}$, comportamiento similar al obtenido en este estudio.

Con la finalidad de identificar los términos que tienen efecto sobre las variables de respuesta (TPC y mangiferina), se utilizó un análisis de varianza (Tabla $1 \mathrm{~S}$, Material Suplementario). En este sentido, se observó que la temperatura $\left(\mathrm{X}_{1}\right)$ y la relación carga/área $\left(\mathrm{X}_{2}\right)$, así como, ambos términos cuadráticos $\left(\mathrm{X}_{1}^{2} \mathrm{y} \mathrm{X}_{2}^{2}\right)$ ejercieron efecto significativo sobre el TPC y concentración de mangiferina. Además, la falta de ajuste no fue significativa $(p>0,05)$, lo que indica que el diseño propuesto es adecuado para describir los datos experimentales. Por otra parte, los coeficientes de determinación señalaron que el $88.2 \%$ y $91.2 \%$ de las variaciones totales producidas en la experimentación, pueden ser explicados por las Ecuaciones 1 y 2 para el TPC y mangiferina, respectivamente:

$T P C=-22.128+0.492 X_{1}+26.394 X_{2}-0.004 X_{1}^{2}-5.728 X_{2}^{2}$

Mangeferina $=-20.129+0.264 X_{1}+13.38 X_{2}-0.002 X_{1}^{2}-$ $2.804 X_{2}^{2}$

En base al modelo generado las condiciones óptimas de extracción fueron $54{ }^{\circ} \mathrm{C}$ y carga/área de $2.3 \mathrm{~kg} \mathrm{~m}^{-2}$, con respuestas predichas de $22.1 \mathrm{mg} \mathrm{GAE} \mathrm{g}^{-1}$ bs y $1.84 \mathrm{mg} \mathrm{g}^{-1}$ bs, para el TPC y mangiferina, respectivamente. Además, para comprobar la validez del modelo matemático, se ensayó la deshidratación de la piel con las condiciones óptimas, con las cuales se obtuvo un TPC de $21.7 \pm 1.6 \mathrm{mg} \mathrm{EAG} \mathrm{g}^{-1}$ bs y $1.94 \pm 0.22 \mathrm{mg} \mathrm{g}^{-1}$ bs de mangiferina. En definitiva, no existió diferencia estadística entre los valores predichos y experimentales, lo que confirma la validez de los modelos encontrados.

Ahora bien, sabiendo que los compuestos fenólicos son termolábiles, ${ }^{26}$ se comparó el efecto de la deshidratación con aire caliente (condiciones óptimas) con el proceso de liofilización

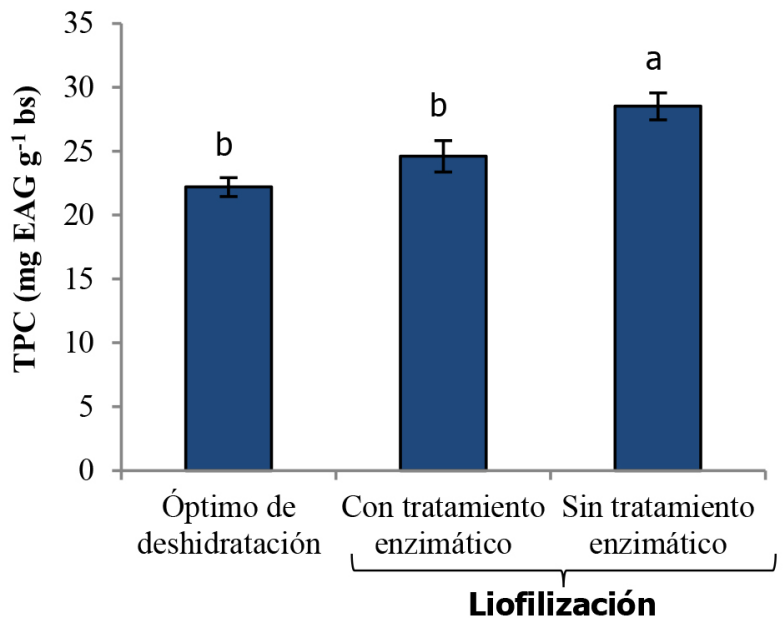

(muestras con y sin tratamiento enzimático) sobre el TPC y concentración de mangiferina de la piel de mango. La liofilización combina el uso de bajas temperatura y presión, lo que permite conservar y retener atributos sensoriales y nutricionales de los alimentos. ${ }^{27}$ Características que hacen que este procedimiento sea ampliamente utilizado frente a la deshidratación con aire caliente, debido a que este último usualmente provoca pérdida de nutrientes. ${ }^{28}$ Como se puede observar en la Figura 2, la deshidratación con aire caliente bajo las condiciones óptimas permite obtener valores estadísticamente similares a los observados con la muestra liofilizada con tratamiento enzimático, convirtiendo a la deshidratación con aire caliente en una opción rentable para el aprovechamiento de este subproducto debido a su bajo costo de equipamiento y operación. ${ }^{29}$ Por otro lado, se observa que el tratamiento enzimático aplicado a la muestra en etapas previas para inactivar enzimas presentes, produce un descenso notorio en el TPC, posiblemente debido a la solubilización inducida de los compuestos durante este proceso. ${ }^{30}$ En contra parte, para mangiferina no se evidenció esta reducción, esto debido a que la mangiferina es escasamente soluble en agua. ${ }^{31}$

\section{Optimización del proceso de extracción}

Una vez optimizado el proceso de deshidratación, se procedió a definir las condiciones de extracción, que nos permitan maximizar la cantidad de compuestos fenólicos extraídos. Como variables independientes se seleccionaron el solvente de extracción ( $\left.\mathrm{Y}_{1}\right)$, tiempo $\left(\mathrm{Y}_{2}\right)$ y temperatura $\left(\mathrm{Y}_{3}\right)$. De igual manera, el TPC y concentración de mangiferina fueron las variables respuesta. Los rangos evaluados se seleccionaron en base resultados previos. Ahora bien, los solventes de extracción se eligieron en función de su seguridad, costo y disponibilidad. En este caso, se utilizó solventes GRAS (generalmente reconocidos como seguros) como el etanol y el agua. Solventes compatibles con la industria alimenticia. Respecto a la temperatura, el valor superior $\left(60^{\circ} \mathrm{C}\right)$ estuvo limitado por la capacidad del equipo de ultrasonidos. Además, Chen, Zhao y $\mathrm{Yu}^{32}$ señalaron que temperaturas superiores a $65{ }^{\circ} \mathrm{C}$, provocan una degradación de los compuestos bioactivos. Finalmente, el tiempo de extracción máximo fue de 120 min, debido a que periodos más prolongados involucran un aumento en los costos de energía y operativos del proceso. ${ }^{33}$

Los resultados alcanzados se muestran en la Tabla 2. Como se puede observar el contenido de fenoles totales varió desde 2.3 hasta $20.0 \mathrm{mg} \mathrm{EAG} \mathrm{g}^{-1}$ bs de piel de mango deshidratada, mientras que la concentración de mangiferina varió desde 0.40 hasta $1.20 \mathrm{mg} \mathrm{g}^{-1} \mathrm{bs}$. Nuevamente, los valores más elevados para las variables analizadas

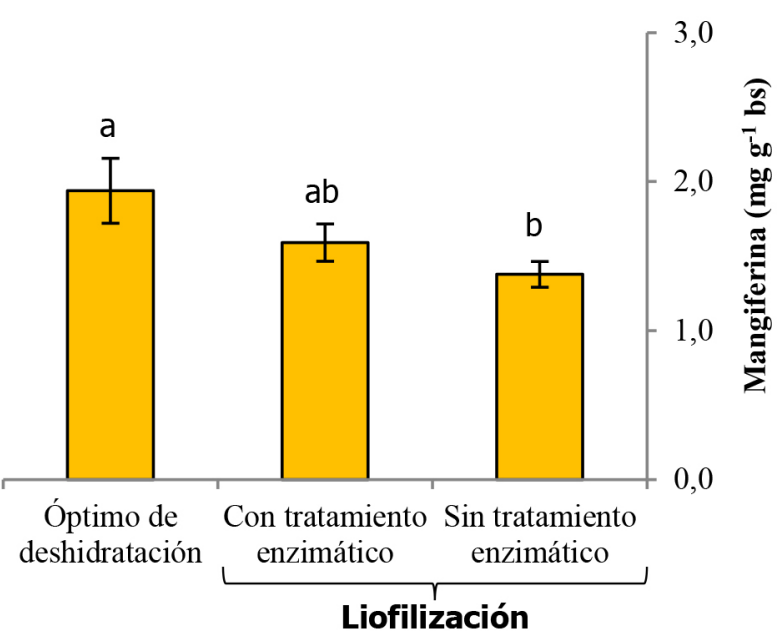

Figura 2. Efecto de los diferentes métodos de deshidratación en el contenido fenólico. Los valores marcados por letras distintas son significativamente diferentes $(p<0,05)$ 
se observaron cerca del punto central del diseño experimental, es decir 50\% EtOH, 62.5 min y $42.5^{\circ} \mathrm{C}$.

La importancia estadística de los factores utilizados se estimó mediante un análisis de varianza (Tabla 2S, Materias Suplementario), encontrando que todos los términos presentaron efecto para el TPC y/o concentración de mangiferina. Se obtuvieron modelos matemáticos cuadráticos completos con un excelente ajuste ( $\mathrm{p}>0.15)$. Además, los coeficientes de determinación indicaron que más del $98 \%$ de las variaciones de las variables están explicadas por el modelo.

Las Ecuaciones 3 y 4 se utilizaron para predecir el TPC y mangiferina, respectivamente.

$T P C=-0.467+0.288 Y_{1}+0.046 Y_{2}+0.468 Y_{3}-0.004 Y_{1}^{2}-0.001 Y_{2}^{2}$ $-0.008 Y_{3}^{2}+0.0003 Y_{1} Y_{2}+0.002 Y_{1} Y_{3}+0.0025 Y_{2} Y_{3}$

Mangiferina $=0.179+0.017 Y_{1}+0.004 Y_{2}+0.019 Y_{3}-0.0002 Y_{1}^{2}-$ $0.00004 Y_{2}^{2}-0.0002 Y_{3}^{2}+0.00003 Y_{1} Y_{2}+0.00004 Y_{1} Y_{3}+$ $0.0001 Y_{2} Y_{3}$

Por otro lado, para evaluar las interacciones entre los diferentes factores estudiados sobre las variables respuesta (fenoles totales y mangiferina), se construyeron las gráficas tridimensionales de superficie correspondientes (Figura 3). Respecto a la interacción solvente y tiempo de extracción, existe un incremento en ambas variables respuesta conforme la concentración de etanol y tiempo de extracción se acercan a 55\% EtOH y 80 min. Sin embargo, al superar estos valores se produce una disminución en las variables de estudio (Figuras 3 a y d). Liu et al.,${ }^{10}$ observaron un comportamiento similar al evaluar las condiciones óptimas para la extracción de compuestos fenólicos presentes en la semilla de Euryale ferox.

Con relación a las variables concentración de etanol y la temperatura de extracción, se puede apreciar que la cantidad de ambas variables respuesta alcanzaron un máximo cuando el solvente utilizado contiene un 55\% EtOH y la temperatura es aproximadamente $55^{\circ} \mathrm{C}$. Al igual que en el caso anterior, es evidente una disminución en las variables, siendo está más marcada para la variable concentración de etanol (Figuras 3 b y e). Este resultado es similar al encontrado en piel de uva por Ghafoor y Choi ${ }^{34}$ quienes observaron un contenido fenólico máximo a $55^{\circ} \mathrm{C}$ y $50 \%$ EtOH.

Finalmente, las Figuras 3 c y f reflejan el efecto del tiempo y la temperatura de extracción, sobre la concentración de fenoles totales y mangiferina. Un incremento en las variables respuesta se alcanza hasta un tiempo de $80 \mathrm{~min}$ y temperatura de extracción de $55^{\circ} \mathrm{C}$. Una vez superados estos límites se produce un descenso en las variables estudiadas. Esta relación también se observó en la determinación de las condiciones óptimas para la extracción de compuestos fenólicos presentes en las hojas de mora. ${ }^{35}$

Las condiciones que permitieron maximizar el contenido fenólico y mangiferina extraídos de la piel de mango fueron $50 \% \mathrm{EtOH}$, 71 min y $51{ }^{\circ} \mathrm{C}$ (Tabla 2). Bajo estos parámetros, se obtuvo un contenido fenólico de $19.5 \pm 0.8 \mathrm{mg} \mathrm{EAG} \mathrm{g}^{-1}$ bs y una concentración de mangiferina de $1.50 \pm 0.13 \mathrm{mg} \mathrm{g}^{-1}$ bs. Los resultados obtenidos experimentalmente no difieren $(\mathrm{p}<0.05)$ con los valores predichos por el modelo (19.37 $\mathrm{mg} \mathrm{EAG} \mathrm{g}^{-1}$ bs y $1.22 \mathrm{mg} \mathrm{g}^{-1} \mathrm{bs}$ ), confirmando que el modelo utilizado fue adecuado para predecir el TPC y concentración de mangiferina.

Con la finalidad de determinar la efectividad de la UAE, se realizó la extracción de los compuestos fenólicos con una técnica convencional (maceración sólido-líquido) desarrollado por PérezJiménez y Saura-Calixto, ${ }^{36}$ método que permite extraer la mayor cantidad de compuestos antioxidantes de matrices vegetales. Sin embargo, con la limitante de utilizar solventes incompatibles con la industria alimentaria como son el metanol y la acetona. En este sentido, con el método tradicional y UAE para el TPC se encontraron los resultados de $22.1 \pm 0.7$ y $19.5 \pm 0.8 \mathrm{mg} \mathrm{GAE} \mathrm{g}^{-1}$ bs, respectivamente. De igual manera, para mangiferina se encontró valores de $1.94 \pm 0.22$ y $1.50 \pm 0.03 \mathrm{mg} \mathrm{g}^{-1}$ bs para los métodos tradicionales y UAE, respectivamente. La ligera diferencia encontrada, se debe al potencial de la UAE, debido principalmente a la cavitación generada cuando una onda ultrasónica se propaga a través de un medio, causando ruptura celular y un aumento en la transferencia de masa. ${ }^{37}$ Lo que le confiere a esta técnica obtener a)

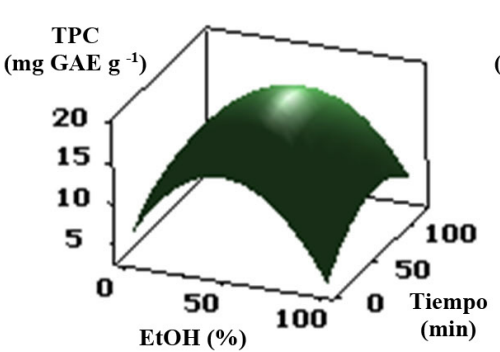

d)

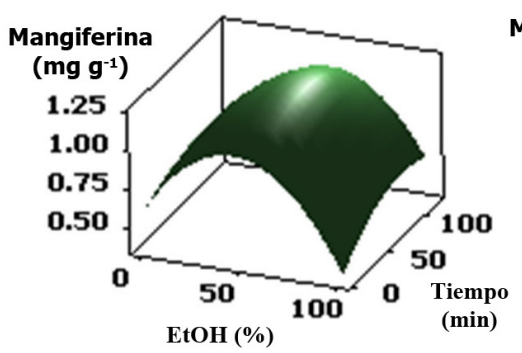

b)

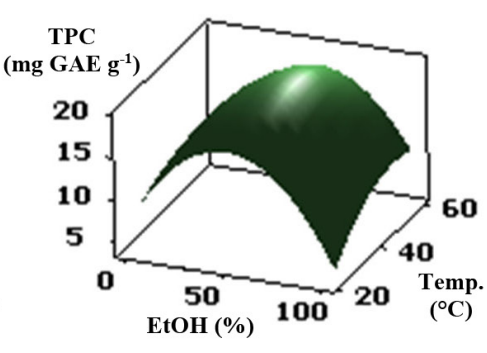

e)

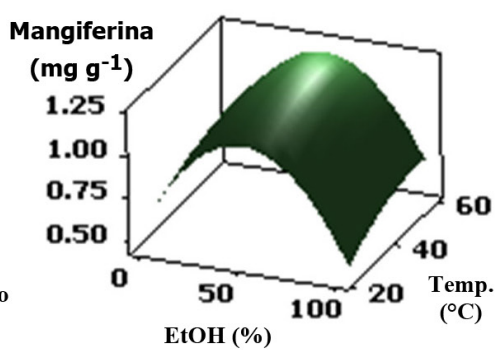

c)

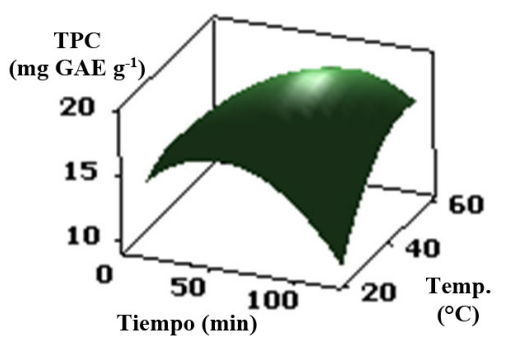

f)

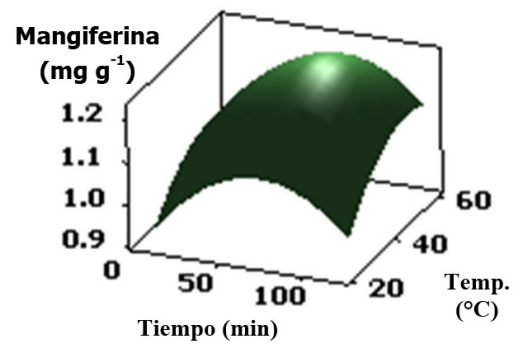

Figura 3. Grafica 3D para la optimización del proceso de extracción: a) efecto de la concentración de etanol y tiempo de extracción sobre el TPC, b) efecto de la concentración de etanol y temperatura de extracción sobre el TPC, c) efecto del tiempo y temperatura de extracción sobre el TPC, d) efecto de la concentración de etanol y tiempo de extracción sobre el contenido de mangiferina, e) efecto de la concentración de etanol y temperatura de extracción sobre el contenido de mangiferina, f) efecto del tiempo y temperatura de extracción sobre el contenido de mangiferina 
un extracto rico en compuestos fenólicos empleando únicamente solventes GRAS. Además, se observó una reducción en el tiempo de extracción cuando se utilizó ultrasonido (71 min), comparado con el tiempo utilizado en la maceración (120 min). Esto demuestra que la UAE es eficiente a la hora de extraer compuestos fenólicos presentes en la piel de mango y permite obtener excelentes resultados en tiempos cortos de extracción.

\section{Identificación de compuestos fenólicos en piel de mango}

La técnica analítica HPLC-ESI-IT-MS ${ }^{\mathrm{n}}$ se utilizó para identificar los compuestos presentes en el extracto obtenido bajo las condiciones óptimas. En este sentido, un total de 38 compuestos se identificaron tentativamente en la piel de mango (Figura 4). Los tiempos de retención, fórmula molecular, $\mathrm{m} / \mathrm{z}$ experimental, espectros de masas, rutas de fragmentación y la información reportada en la literatura, fueron indispensables para la identificación de compuestos, esta información se detalla en la Tabla 3. Los compuestos detectados permanecen a diversas familias como: ácidos orgánicos, ácidos fenólicos, galatos y galatoninos, flavonoides, xantonas y derivados de la benzofenona.

\section{Ácidos orgánicos}

Cuatro ácidos orgánicos fueron identificados. El ácido málico $(\operatorname{Tr}=1.42 \mathrm{~min}$ ) exhibió un ion con $\mathrm{m} / \mathrm{z} 133$ y su espectro MS/MS mostró un fragmento a m/z 115 correspondiente [M-H-H2O]-. ${ }^{39}$ Además, se detectaron tres compuestos con ion m/z 191, los cuales fueron identificados tentativamente como ácido quínico, ácido cítrico y ácido isocítrico, en base a su orden de elución comparados con estudios previos. ${ }^{39}$

\section{Flavonoides}

Respecto a la familia de los flavonoides, nuevos compuestos fueron identificados. En este sentido, el pico $42(\mathrm{Tr}=23.79 \mathrm{~min})$ presentó un ion con $\mathrm{m} / \mathrm{z}$ de 301 y un fragmento principal a m/z 151 debido a una reacción inversa de Diels Alder (RDA). En base a esta información se identificó como quercetina. ${ }^{39}$ Además, cinco derivados de la quercetina fueran detectados e identificados como quercetina3-O-diglicósido $(\mathrm{Tr}=15.18 \mathrm{~min})$, quercetina-3-O-glucósido $(\operatorname{Tr}=16.72 \mathrm{~min})$, quercetina-3-O-galactósido $(\mathrm{Tr}=17.02 \mathrm{~min})$, pentosido de quercetina $(\mathrm{Tr}=18.37 \mathrm{~min})$ y quercetina-3-O-ramnosida
( $\operatorname{Tr}=18.81 \mathrm{~min})$. La identificación se realizó en base a los iones moleculares y el fragmento con $\mathrm{m} / \mathrm{z}=301$ característico de la aglicona, que presentaron todos estos compuestos. Cabe indicar, que los isómeros pico 27 y 29, fueron identificados en base al orden de elución detallado por. ${ }^{40}$ Por otra parte, los picos 38 y 39 se identificaron como Ramnetina-3-[6"-2 butenoil-hexósido] con un ion a m/z 545 y tiempos de retención de 19.15 y $19.51 \mathrm{~min}$, respectivamente. El perfil de fragmentación mostró un ion a m/z 469, coincidiendo con la información reportada por Dorta et al. ${ }^{40}$

Finalmente, el pico $41(\mathrm{Tr}=23,07 \mathrm{~min})$ exhibió un ion a $\mathrm{m} / \mathrm{z} 477.65$, y un fragmento a $\mathrm{m} / \mathrm{z}=315$ correspondiente a la aglicona ramnetina. De acuerdo con la información reportada por Dorta et al., ${ }^{40}$ este compuesto se identificó tentativamente como ramnetina 3-O-hexósido.

\section{Galatos y galatoninos}

La evaluación del cromatograma (Figura 4) obtenido permitió identificar 11 compuestos pertenecientes a la familia de galatos y galatoninos. El pico $5(\mathrm{Tr}=2.27 \mathrm{~min}) \mathrm{a} \mathrm{m} / \mathrm{z} 331$ y con fórmula molecular $\mathrm{C}_{13} \mathrm{H}_{16} \mathrm{O}_{10}$ se identificó tentativamente como glucogalina. El modo MS/MS reflejó un fragmento común a m/z 169, causado por la presencia de una fracción de ácido gálico, producto de la pérdida de una molécula de glucosa. ${ }^{19}$ Así mismo, los picos 6 y 9, cuyos tiempos de retención fueron 2.89 y $5.53 \mathrm{~min}$, respectivamente, presentaron un ion molecular y un espectro MS/MS similar al compuesto antes mencionado, por lo tanto, se consideraron isómeros de la glucogalina. Adicionalmente, el galato conocido como ácido gálico (pico 8) también fue detectado, gracias a su ion a m/z 169 y su fragmento característico (m/z 125) producido por la pérdida de una molécula de $\mathrm{CO}_{2}{ }^{40}$ Además, el pico 8 se identificó como ácido 5-galoilquínico con un ion a $\mathrm{m} / \mathrm{z}$ 343 y un tiempo de retención de $4.03 \mathrm{~min}$. El perfil de fragmentación mostró un ion a m/z 169, seguido por otro a m/z 191, coincidiendo con la información reportada por López-Cobo et al. ${ }^{38}$ Por otra parte, el galato de metilo $(\mathrm{Tr}=9.24 \mathrm{~min})$ exhibió un ion a $\mathrm{m} / \mathrm{z} 183$ y su espectro MS/MS mostró un fragmento a m/z 168, provocado por la pérdida de $-\mathrm{CO}_{2} \mathrm{CH}_{3}{ }^{40}$ Por otra parte, el pico $13(\mathrm{Tr}=9.44 \mathrm{~min})$, presentó un ion molecular a m/z 495 y un espectro MS/MS con fragmentos 191 y 343, fue identificado como ácido 3,5 digaloilquínico, en concordancia con el estudio de Clifford, Stoupi y Kuhnert. ${ }^{42}$

Los picos 31 y 34, presentaron el mismo ion molecular a m/z 335 y fragmento MS/MS 183, correspondiente a un galato de metilo,

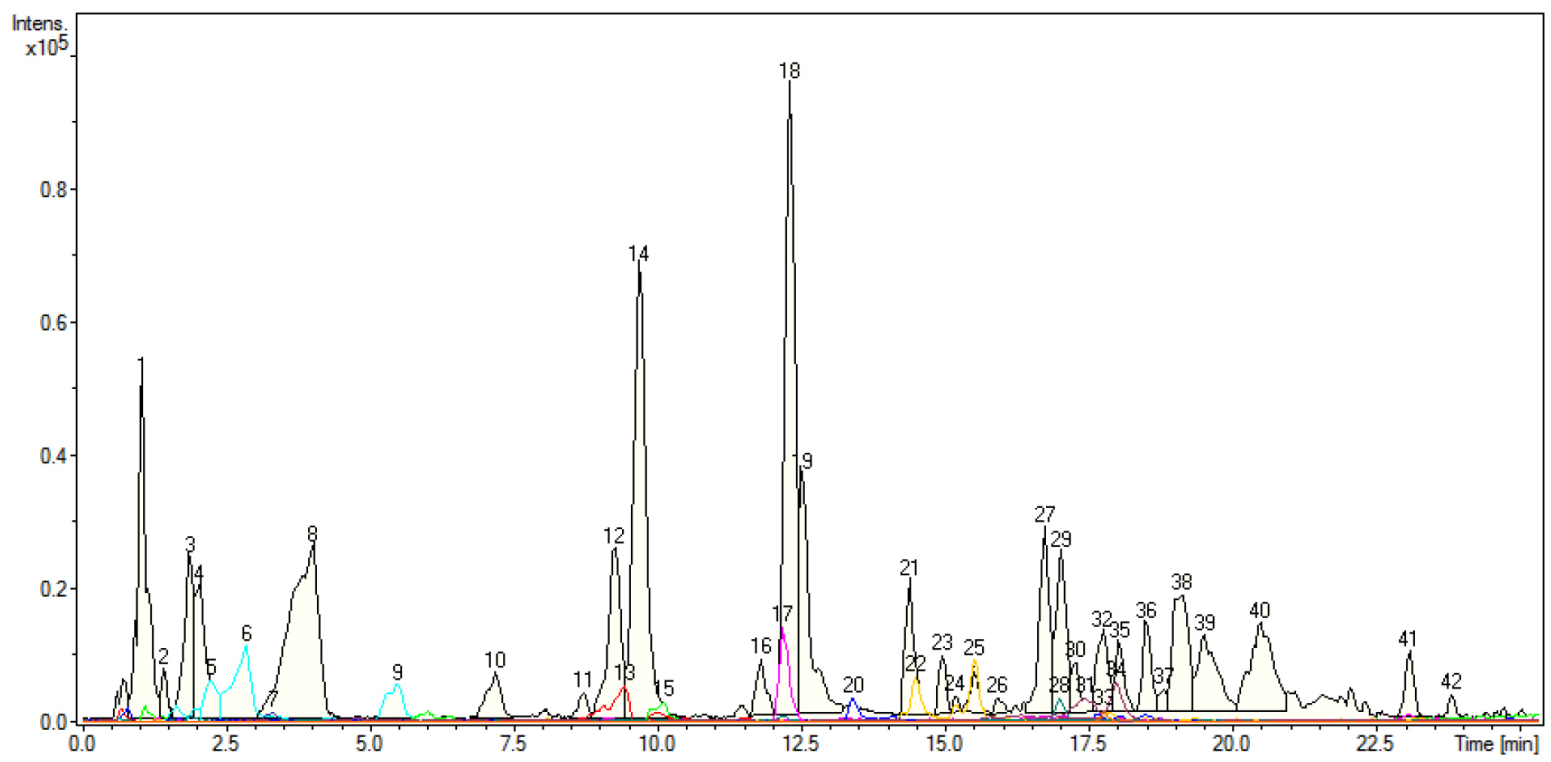

Figura 4. Cromatograma del extracto de piel de aguacate obtenido por HPLC-ESI-IT-MS ${ }^{n}$ 
Tabla 3. Identificación de compuestos en el extracto de cáscara de mango por HPLC-ESI-IT-MS ${ }^{\mathrm{n}}$

\begin{tabular}{|c|c|c|c|c|c|c|}
\hline \# & $\operatorname{Tr}(\min )$ & $\begin{array}{l}\text { Formula mo- } \\
\text { lecular }\end{array}$ & $\mathrm{m} / \mathrm{z}$ & Fragmentos MS/MS & Identificación tentativa & \\
\hline 1 & 1.06 & $\mathrm{C}_{7} \mathrm{H}_{12} \mathrm{O}_{6}$ & 191 & n.f. & Ácido quínico & $*$ \\
\hline 2 & 1.42 & $\mathrm{C}_{4} \mathrm{H}_{6} \mathrm{O}_{5}$ & 133 & 115 & Ácido málico & $\bullet$ \\
\hline 3 & 1.87 & $\mathrm{C}_{6} \mathrm{H}_{8} \mathrm{O}_{7}$ & 191 & 111,173 & Ácido cítrico & $\bullet$ \\
\hline 4 & 2.07 & $\mathrm{C}_{6} \mathrm{H}_{8} \mathrm{O}_{7}$ & 191 & 111,173 & Ácido isocítrico & $\bullet$ \\
\hline 5 & 2.27 & $\mathrm{C}_{13} \mathrm{H}_{16} \mathrm{O}_{10}$ & 331 & $125,169,211,241,271$ & Glucogalina & $\bullet$ \\
\hline 6 & 289 & $\mathrm{C}_{13} \mathrm{H}_{16} \mathrm{O}_{10}$ & 331 & $169,211,271$ & Glucogalina & $\bullet$ \\
\hline 7 & 3.33 & $\mathrm{C}_{7} \mathrm{H}_{6} \mathrm{O}_{5}$ & 169 & 125 & Ácido gálico & $\boldsymbol{\Delta}$ \\
\hline 8 & 4.03 & $\mathrm{C}_{14} \mathrm{H}_{16} \mathrm{O}_{10}$ & 343 & 169,191 & Ácido galoilquínico & $\bullet$ \\
\hline 9 & 5.53 & $\mathrm{C}_{13} \mathrm{H}_{16} \mathrm{O}_{10}$ & 331 & 169 & Glucogalina & $\bullet$ \\
\hline 10 & 7.20 & $\mathrm{C}_{19} \mathrm{H}_{20} \mathrm{O}_{11}$ & 423 & 303 & Maclurin-3-C- $\beta$-D-glucósido & $\Delta$ \\
\hline 11 & 8.72 & $\mathrm{C}_{14} \mathrm{H}_{18} \mathrm{O}_{9}$ & 329 & 209,239 & Glucósido de ácido vanílico & $*$ \\
\hline 12 & 9.24 & $\mathrm{C}_{8} \mathrm{H}_{8} \mathrm{O}_{5}$ & 183 & 124 & Galato de metilo & $\Delta$ \\
\hline 13 & 9.44 & $\mathrm{C}_{21} \mathrm{H}_{20} \mathrm{O}_{14}$ & 495 & 191,343 & Ácido 3,5 digaloilquínico & $*$ \\
\hline 14 & 9.68 & $\mathrm{C}_{26} \mathrm{H}_{24} \mathrm{O}_{15}$ & 575 & $303,333,405,423$ & Maclurin-3-C-(2-O-galloil)- $\beta$-D-glucósido & $\boldsymbol{\Delta}$ \\
\hline 15 & 10.11 & $\mathrm{C}_{15} \mathrm{H}_{18} \mathrm{O}_{8}$ & 325 & 163 & Glucósido de ácido cumarico & * \\
\hline 16 & 11.80 & & 553 & 385,517 & & \\
\hline 17 & 12.21 & $\mathrm{C}_{26} \mathrm{H}_{24} \mathrm{O}_{14}$ & 559 & $287,317,389,407$ & Iriflofenona 3-C-(2-O-galloil)- $\beta$-D-glucósido & $\Delta$ \\
\hline 18 & 12.30 & $\mathrm{C}_{19} \mathrm{H}_{18} \mathrm{O}_{11}$ & 421 & 301,331 & Mangiferina & $\Delta$ \\
\hline 19 & 12.49 & $\mathrm{C}_{33} \mathrm{H}_{28} \mathrm{O}_{19}$ & 727 & 557,575 & Maclurin-3-C- (2,3-di-O-galloil)- $\beta$-D-glucósido & $\Delta$ \\
\hline 20 & 13.42 & $\mathrm{C}_{20} \mathrm{H}_{20} \mathrm{O}_{11}$ & 435 & 315,345 & Metoxi-mangiferina & $*$ \\
\hline 21 & 14.40 & & 543 & $285,327,405$ & & \\
\hline 22 & 14.54 & $\mathrm{C}_{26} \mathrm{H}_{22} \mathrm{O}_{15}$ & 573 & 421 & Galato de mangiferina & $\Delta$ \\
\hline 23 & 14.95 & $\mathrm{C}_{33} \mathrm{H}_{28} \mathrm{O}_{18}$ & 711 & $389,407,541,559$ & Iriflofenona 3-C-(2",6"-di-O-galloil)- $\beta$-D-glucósido & $\boldsymbol{\Delta}$ \\
\hline 24 & 15.18 & $\mathrm{C}_{26} \mathrm{H}_{28} \mathrm{O}_{16}$ & 595 & 301 & Quercetina-3-O-diglicósido & $\bullet$ \\
\hline 25 & 15.52 & $\mathrm{C}_{26} \mathrm{H}_{22} \mathrm{O}_{15}$ & 573 & 421 & Galato de mangiferina & $\Delta$ \\
\hline 26 & 15.91 & $\mathrm{C}_{34} \mathrm{H}_{28} \mathrm{O}_{22}$ & 787 & n.f. & Tetragaloil-glucosa & $\Delta$ \\
\hline 27 & 16.72 & $\mathrm{C}_{21} \mathrm{H}_{20} \mathrm{O}_{12}$ & 463 & 301 & Quercetina-3-O-glucósido & $\bullet$ \\
\hline 28 & 16.97 & $\mathrm{C}_{26} \mathrm{H}_{32} \mathrm{O}_{16}$ & 599 & n.f. & Dímero de glucósido del ácido p-hidroxibenzoico & $\bullet$ \\
\hline 29 & 17.02 & $\mathrm{C}_{21} \mathrm{H}_{20} \mathrm{O}_{12}$ & 463 & 301 & Quercetina-3-O-galactósido & $\bullet$ \\
\hline 30 & 17.28 & & 695 & $285,327,405,557,634$ & & \\
\hline 31 & 17.43 & $\mathrm{C}_{15} \mathrm{H}_{12} \mathrm{O}_{9}$ & 335 & 183 & Éster de galato de metilo & $\bullet$ \\
\hline 32 & 17.76 & $\mathrm{C}_{24} \mathrm{H}_{22} \mathrm{O}_{10}$ & 469 & 301 & Dilactona del ácido valoneico & $\bullet$ \\
\hline 33 & 17.76 & $\mathrm{C}_{41} \mathrm{H}_{32} \mathrm{O}_{26}$ & 939 & $\mathrm{n}, \mathrm{f}$ & Pentagaloil-glucosa & $\Delta$ \\
\hline 34 & 17.91 & $\mathrm{C}_{15} \mathrm{H}_{12} \mathrm{O}_{9}$ & 335 & 183 & Éster de galato de metilo & $\bullet$ \\
\hline 35 & 18.37 & $\mathrm{C}_{20} \mathrm{H}_{18} \mathrm{O}_{11}$ & 433 & 301 & Pentosida de quercetina & $\Delta$ \\
\hline 36 & 18.47 & $\mathrm{C}_{24} \mathrm{H}_{22} \mathrm{O}_{10}$ & 469 & 301 & Dilactona del ácido valoneico & $\bullet$ \\
\hline 37 & 18.81 & $\mathrm{C}_{21} \mathrm{H}_{20} \mathrm{O}_{11}$ & 447 & 301 & Quercetina-3-O-ramnosida (quercitrina) & $\bullet$ \\
\hline 38 & 19.15 & $\mathrm{C}_{26} \mathrm{H}_{16} \mathrm{O}_{13}$ & 545 & 469 & Ramnetina-3-[6"-2 butenoil-hexósido] & $\bullet$ \\
\hline 39 & 19.51 & $\mathrm{C}_{26} \mathrm{H}_{16} \mathrm{O}_{13}$ & 545 & 469 & Ramnetina-3-[6"-2 butenoil-hexósido] & $\bullet$ \\
\hline 40 & 20.49 & & 621 & 469,545 & & \\
\hline 41 & 23.21 & $\mathrm{C}_{22} \mathrm{H}_{22} \mathrm{O}_{12}$ & 477 & 315 & Ramnetina 3-O-hexósido & • \\
\hline 42 & 23.79 & $\mathrm{C}_{15} \mathrm{H}_{10} \mathrm{O}_{7}$ & 301 & 151,179 & Quercetina & $\bullet$ \\
\hline
\end{tabular}

Fuente: * López-Cobo et al.,$^{38} \bullet$ Figueroa et al. ${ }^{39} \bullet$ Dorta et al.,${ }^{40} \boldsymbol{\Delta}$ Barreto et al. ${ }^{41}$

fueron identificados como isómeros del éster de galato de metilo. ${ }^{40}$ Finalmente, los picos 26 y 33, fueron identificados como tetragaloilglucosa y pentagaloil-glucosa, en función a los iones moleculares detectados. Estos compuestos fueron previamente reportados por Dorta et al. ${ }^{40}$ y López-Cobo et al. ${ }^{38}$

\section{Derivados de la benzofenona}

Un total de 5 derivados de la benzofenona fueron detectados por la metodología utilizada en el presente estudio. El pico 10 ( $\mathrm{Tr}=7.10 \mathrm{~min}$ ) presentaron un ion a $\mathrm{m} / \mathrm{z} 423$, mientras que su patrón de fragmentación reflejó un ion a m/z 303 provocado por la escisión del 
C-glucósido, ${ }^{41}$ fue identificado como Maclurin-3-C- $\beta$-D-glucósido.

Por otro lado, los picos 14, 17, 19 y 23 con iones moleculares de 575, 559, 727 y 711, respectivamente. Todos presentaron en sus espectros MS/MS fragmentos por la pérdida de 152 Da y $170 \mathrm{Da}$, indicando la presencia de un grupo galoil y ácido gálico. Además, se presentaron los fragmentos $333,317,485$ y 469 , respectivamente, debido a escisión del C-glucósido (90 Da) y eliminación de grupo galoil (152 Da). En base a esta información los picos fueron identificados como maclurin-3-C-(2-O-galloil)- $\beta$-D-glucósido, iriflofenona 3-C-(2-O-galloil)- $\beta$-D-glucósido, maclurin-3-C-(2,3di-O-galloil)- $\beta$-D-glucósido y iriflofenona 3-C-(2",6"-di-O-galloil)$\beta$-D-glucósido, respectivamente.

\section{Xantonas y sus derivados}

En la actualidad, los compuestos fenólicos pertenecientes a la familia de las xantonas juegan un papel indispensable en la industria alimentaria, pues gracias a sus poderosas propiedades las convierte en un antioxidante más potente que la vitamina $\mathrm{C}$ y la vitamina $\mathrm{E}^{1,40}$

Los picos $18(\mathrm{Tr}=12.30 \mathrm{~min})$ y $20(\mathrm{Tr}=13.42 \mathrm{~min})$ con fórmulas $\mathrm{C}_{19} \mathrm{H}_{18} \mathrm{O}_{11}$ y $\mathrm{C}_{20} \mathrm{H}_{20} \mathrm{O}_{11}$ con iones a m/z 421 y 435 . Presentaron un patrón fragmentación similar $(\mathrm{M}-\mathrm{H}-120 \mathrm{Da})$ y ( $\mathrm{M}-\mathrm{H}-90 \mathrm{Da})$, derivados de la escisión del C-glucósido. ${ }^{41}$ Este patrón de fragmentación corresponde a los compuestos mangiferina y metoxi-mangiferina, respectivamente.

Por otro lado, los picos 22 y 25 exhibieron un ion molecular a m/z 573 y un fragmento predominante a m/z 421 debido a eliminación de grupo galoil (152 Da). Por lo tanto, estos compuestos fueron identificados como isómeros del galato de mangiferina. La presencia de estos compuestos coincidieron con la información reportada por Barreto et al., ${ }^{41}$ Dorta et al..${ }^{40}$ y Gómez-Caravaca et al.. ${ }^{19}$

\section{Otros compuestos polares}

Adicionalmente, el análisis cromatográfico reveló la presencia de compuestos fenólicos pertenecientes a la familia de ácidos hidroxicinámicos e hidroxibenzoicos. En este sentido, el pico 11 ( $\mathrm{Tr}=8.72 \mathrm{~min}$ ) se identificó como glucósido de ácido vanílico, con un espectro MS/MS con fragmentos a m/z 209 (M - H - 120 Da) y 239 ( $\mathrm{M}-\mathrm{H}-90 \mathrm{Da})$, derivados de la escisión del C-glucósido. El pico 15 $(\mathrm{Tr}=10.11 \mathrm{~min})$ con ion molecular a m/z 325 y fragmento a m/z 163, producto de la pérdida de una molécula de glucosa, fue identificado como glucósido de ácido cumárico. Por otra parte, el pico 28 fue identificado como dímero de glucósido del ácido p-hidroxibenzoico en función a su m/z 559, coincidiendo con la información reportada por Gómez-Caravaca et al. ${ }^{19}$

Finalmente, dos compuestos derivados del ácido elágico se detectaron. Los picos 32 y 36, ambos con ion molecular 469 y fragmento a 301 , fueron identificados como isómeros dilactona del ácido valoneico. ${ }^{40}$

\section{CONCLUSIONES}

Los procesos de deshidratación y de extracción de compuestos fenólicos en piel de mango fueron optimizados de manera exitosa mediante la metodología de superficie de respuesta. Para el proceso de deshidratación, las condiciones óptimas fueron $54{ }^{\circ} \mathrm{C}$ y $2.3 \mathrm{~kg} \mathrm{~m}^{-2}$. Bajo estos parámetros se obtuvo un TPC de $21.7 \pm 1.6 \mathrm{mg} \mathrm{EAG} \mathrm{g}^{-1}$ bs y, una concentración de mangiferina de $1.94 \pm 0.22 \mathrm{mg} \mathrm{g}^{-1} \mathrm{bs}$. Por otro lado, las condiciones óptimas para la UAE fueron $50 \%$ EtOH, $71 \mathrm{~min}$ y $51{ }^{\circ} \mathrm{C}$, con estas condiciones se encontró un TPC $19.5 \pm 0.8 \mathrm{mg} \mathrm{EAG} \mathrm{g}^{-1}$ bs y un contenido de mangiferina de $1.50 \pm 0.13 \mathrm{mg} \mathrm{g}^{-1} \mathrm{bs}$. Finalmente, en el extracto obtenido con las condiciones óptimas, se logró identificar 38 compuestos mediante la técnica de HPLC-ESI-IT-MS ${ }^{\mathrm{n}}$.

\section{MATERIAL SUPLEMENTARIO}

Los datos del ANOVA están disponibles en http://quimicanova. sbq.org.br, en formato pdf, con acceso gratuito.

\section{AGRADECIMIENTOS}

Los autores agradecen a la Universidad Técnica Particular de Loja (UTPL) por la financiación del estudio. Agradecen también a los revisores pares y a los editores de esta revista por sus comentarios, que ayudaron a mejorar este trabajo.

\section{REFERENCIAS}

1. Martin, M.; He, Q.; Food Rev. Int. 2009, 25, 346.

2. Singh, Z.; Singh, R. K.; Sane, V. A.; Nath, P.; CRC. Crit. Rev. Plant Sci. 2013, 32, 217.

3. Kim, H.; Moon, J. Y.; Kim, H.; Lee, D.-S.; Cho, M.; Choi, H.-K.; Kim, Y. S.; Mosaddik, A.; Cho, S. K.; Food Chem. 2010, 121, 429.

4. Dorta, E.; Lobo, M. G.; González, M.; J. Food Sci. 2012, 77, 80.

5. Sogi, D. S.; Siddiq, M.; Greiby, I.; Dolan, K. D.; Food Chem. 2013, 141, 2649.

6. Sogi, D. S.; Siddiq, M.; Roidoung, S.; Dolan, K. D.; J. Food Sci. 2012, 77, 1197.

7. Figueroa, J. G.; Borrás-Linares, I.; Lozano-Sánchez, J.; Quirantes-Piné, R.; Segura-Carretero, A.; Electrophoresis 2018, 39, 1908.

8. Tiwari, B. K.; Trends Anal. Chem. 2015, 71, 100.

9. Şahin, S.; Aybastier, Ö.; Işik, E.; Food Chem. 2013, 141, 1361.

10. Liu, Y.; Wei, S.; Liao, M.; Ind. Crops Prod. 2013, 49, 837.

11. Bashi, D.; Mortazavi, S. A.; Rezaei, K.; Rajaei, A.; Karimkhani, M. M.; Food Sci. Biotechnol. 2012, 21, 1005.

12. Sun, Y.; Liu, J.; Kennedy, J. F.; Carbohydr. Polym. 2010, 80, 949.

13. Mendoza, R.; Herrera, A. O.; Inf. Tecnol. 2012, 23, 73.

14. Horwitz, W.; Official Methods of Analysis of AOAC International; $16^{\text {th }}$ ed.; AOAC International.: Washington, 2010.

15. Nasirizadeh, N.; Dehghanizadeh, H.; Yazdanshenas, M. E.; Moghadam, M. R.; Karimi, A.; Ind. Crops Prod. 2012, 40, 361.

16. Armijos, M. A.; Figueroa, J. G.; Revista Venezolana de Ciencia y Tecnología de Alimentos 2012, 3, 202.

17. Pérez-Jiménez, J.; Saura-Calixto, F.; J. Agric. Food Chem. 2005, 53, 5036.

18. Bezerra, M. A.; Santelli, R. E.; Oliveira, E. P.; Villar, L. S.; Escaleira, L. A.; Talanta 2008, 76, 965.

19. Gómez-Caravaca, A. M.; López-Cobo, A.; Verardo, V.; Segura-Carretero, A.; Fernández-Gutiérrez, A.; Electrophoresis 2016, 37, 1072.

20. Dong, X.; Hu, Y.; Li, Y.; Zhou, Z.; Sci. Hortic. (Amsterdam). 2019, 243, 281.

21. Oliveira, B. G.; Costa, H. B.; Ventura, J. A.; Kondratyuk, T. P.; Barroso, M. E. S.; Correia, R. M.; Pimentel, E. F.; Pinto, F. E.; Endringer, D. C.; Romão, W.; Food Chem. 2016, 204, 37.

22. Saavedra, J.; Córdova, A.; Navarro, R.; Díaz-Calderón, P.; Fuentealba, C.; Astudillo-Castro, C.; Toledo, L.; Enrione, J.; Galvez, L.; J. Food Eng. 2017, 198, 81.

23. Lang, G. H.; Lindemann, I. da S.; Ferreira, C. D.; Hoffmann, J. F.; Vanier, N. L.; de Oliveira, M.; Food Chem. 2019, 287, 197.

24. Gümüşay, Ö. A.; Borazan, A. A.; Ercal, N.; Demirkol, O.; Food Chem. $\mathbf{2 0 1 5}, 173,156$.

25. Miranda, M.; Vega-Gálvez, A.; López, J.; Parada, G.; Sanders, M.; Aranda, M.; Uribe, E.; Di Scala, K.; Ind. Crops Prod. 2010, 32, 258.

26. Berardini, N.; Knödler, M.; Schieber, A.; Carle, R.; Innovative Food Sci. Emerging Technol. 2005, 6, 442.

27. Valadez-Carmona, L.; Plazola-Jacinto, C. P.; Hernández-Ortega, M.; Hernández-Navarro, M. D.; Villarreal, F.; Necoechea-Mondragón, H.; 
Ortiz-Moreno, A.; Ceballos-Reyes, G.; Innovative Food Sci. Emerging Technol. 2017, 41, 378.

28. de Ancos, B.; Sánchez-Moreno, C.; Zacarías, L.; Rodrigo, M. J.; Sáyago Ayerdí, S.; Blancas Benítez, F. J.; Domínguez Avila, J. A.; GonzálezAguilar, G. A.; J. Food Meas. Charact. 2018, 12, 2145.

29. Tello-Ireland, C.; Lemus-Mondaca, R.; Vega-Gálvez, A.; López, J.; Di Scala, K.; LWT - Food Sci. Technol. 2011, 44, 2112.

30. Amin, I.; Norazaidah, Y.; Hainida, K. I. E.; Food Chem. 2006, 94, 47.

31. Ochocka, R.; Hering, A.; Stefanowicz-Hajduk, J.; Cal, K.; Barańska, H.; PLoS One 2017, 12, 1 .

32. Chen, M.; Zhao, Y.; Yu, S.; Food Chem. 2014, 172, 543.

33. Tomšik, A.; Pavlić, B.; Vladić, J.; Ramić, M.; Brindza, J.; Vidović, S.; Ultrason. Sonochem. 2016, 29, 502.

34. Ghafoor, K.; Choi, Y. H.; J. Korean Soc. Appl. Biol. Chem. 2009, 52, 295.

35. Aybastier, Ö.; Işik, E.; Şahin, S.; Demir, C.; Ind. Crops Prod. 2013, 44, 558.
36. Pérez-Jiménez, J.; Saura-Calixto, F.; V Congreso Iberoamericano de Tecnología Postcosecha y Agroexportaciones 2007, 1150.

37. Wang, J.; Sun, B.; Cao, Y.; Tian, Y.; Li, X.; Food Chem. 2008, 106, 804.

38. López-Cobo, A.; Gómez-Caravaca, A. M.; Pasini, F.; Caboni, M. F.; Segura-Carretero, A.; Fernández-Gutiérrez, A.; LWT - Food Sci. Technol. 2016, 73, 505.

39. Figueroa, J. G.; Borrás-Linares, I.; Lozano-Sánchez, J.; SeguraCarretero, A.; Food Res. Int. 2018, 105, 752.

40. Dorta, E.; González, M.; Lobo, M. G.; Sánchez-Moreno, C.; de Ancos, B.; Food Res. Int. 2014, 57, 51.

41. Barreto, J.; Trevisan, M. T. .; Hull, W.; Erben, G.; de Brito, E.; Pfundstein, B.; Würtele, G.; Spiegelhalder, B.; Owen, R.; J. Agric. Food Chem. 2008, 56, 5599.

42. Clifford, M. N.; Stoupi, S.; Kuhnert, N.; J. Agric. Food Chem. 2007, 55, 2797. 